\title{
Cyclin A overexpression is associated with chemosensitivity to paclitaxel-based chemotherapy in patients with esophageal squamous cell carcinoma
}

\author{
JUN-XING HUANG ${ }^{1}$, SHUANG-LE SHEN ${ }^{1}$, MEI LIN ${ }^{1}$, WEI XIAO ${ }^{2}$, WEI-CHANG CHEN ${ }^{3}$, \\ MAO-SONG LIN ${ }^{1}$, HONG YU ${ }^{2}$, PING CHEN ${ }^{2}$ and RONG-YU QIAN ${ }^{1}$ \\ Departments of ${ }^{1}$ Oncology and ${ }^{2}$ Pathology, The People's Hospital of Taizhou, Taizhou Medical School, \\ Nantong and Yangzhou University, Taizhou, Jiangsu 225300; ${ }^{3}$ Department of Gastroenterology, The \\ First Affiliated Hospital of Soochow University, Suzhou, Jiangsu 215006, P.R. China
}

Received April 6, 2012; Accepted July 12, 2012

DOI: $10.3892 / \mathrm{ol} .2012 .814$

\begin{abstract}
The aim of this study was to investigate the correlation between cyclin A expression and efficacy of paclitaxel-based chemotherapy in patients with esophageal squamous cell carcinoma (ESCC). The expression of cyclin A was examined in 48 newly diagnosed ESCC patients prior to treatment using the MaxVision immunohistochemistry method. The patients received four cycles of paclitaxel-based chemotherapy, the short-term treatment efficacy was evaluated and a 3-year follow-up was conducted. The response rate was greater in patients with positive cyclin A expression compared with those with negative expression ( 54.8 vs. $\left.23.5 \% ; \chi^{2}=4.373 ; \mathrm{P}<0.05\right)$. Univariate and multivariate Cox analysis revealed that clinicopathological stage, degree of differentiation and expression of cyclin A were independent prognosis factors in patients with ESCC following paclitaxel-based chemotherapy. ESCC patients with positive cyclin A expression demonstrated an increased sensitivity to paclitaxel-based chemotherapy, suggesting that cyclin A may be used as a marker to predict the treatment efficacy of paclitaxel in patients with ESCC.
\end{abstract}

\section{Introduction}

Esophageal squamous cell carcinoma (ESCC) remains the most common type of esophageal cancer worldwide. Even in resectable esophageal cancer, the 5-year survival rate remains only $14-45 \%(1,2)$. Treatment failure of ESCC is due to the

Correspondence to: Dr Jun-Xing Huang, Department of Oncology, The People's Hospital of Taizhou, Taizhou Medical School, Nantong and Yangzhou University, No. 210 Yingchun Road, Taizhou, Jiangsu 225300, P.R. China

E-mail: huangjunxing@yahoo.com.cn

Key words: esophageal squamous cell carcinoma, cyclin A, paclitaxel, immunohistochemistry, chemotherapy high incidence of local-regional failure and early systemic dissemination of the disease (3). Chemotherapy is one of the most important treatment methods for advanced esophageal carcinoma. Several prospective and retrospective studies in patients with locally advanced thoracic esophageal cancers or in elderly patients with early stage esophageal cancer, particularly epidermoid, who respond to chemoradiotherapy, have suggested that there is no benefit from the addition of surgery following chemoradiotherapy compared with the continuation of additional chemoradiotherapy. Following chemoradiotherapy, with salvage therapy if required, treatment outcomes among patients with resectable thoracic ESCC were comparable or superior to the treatment outcomes following surgery alone (4-7). Therefore, the role of chemotherapy is becoming increasingly important in the treatment of ESCC.

Patients with ESCC demonstrate different sensitivity for chemotherapy. Therefore, in order to improve the prognosis of esophageal carcinoma, there is an urgent requirement to predict the sensitivity of chemotherapy drugs and tailor individual treatment. Paclitaxel is one of the most effective drugs for esophageal carcinoma treatment as it targets cells in the $\mathrm{G} 2 / \mathrm{M}$ phase transition. As a critical regulatory protein, cyclin A may promote $\mathrm{G} 2 / \mathrm{M}$ phase transition and mitosis (8). Our previous studies have revealed that overexpression of cyclin A in ESCC is associated with cell proliferation and survival (9-11). As the clinical application of immunohistochemistry is relatively cost-effective (2), in this study, we explore the possibility of evaluating paclitaxel efficacy by examining the correlation between cyclin A expression and efficacy of paclitaxel-based chemotherapy in patients with ESCC.

\section{Patients and methods}

Patients and specimens. All specimens collected for this retrospective study were obtained from patients who had not received chemotherapy or radiotherapy prior to biopsy. A total of 48 ESCC patients were diagnosed by endoscopic biopsy pathology between 2006 and 2009 at The People's Hospital of Taizhou (Taizhou, Jiangsu, China). Those deemed 
unsuitable for surgery based on their performance status, bulky local disease, Karnofsky score ( $\geq 70$ points) or personal choice received chemotherapy. Of the 48 patients, 33 were male and 15 were female. The age of the patients ranged from 47 to 78 years and the median age was 58 years. A total of 16, 19 and 13 cases were upper, middle and lower thoracic ESCC, respectively. Staging was determined according to the American Joint Committee on Cancer tumor-node-metastasis (TNM) classification (12). A total of 7, 13 and 28 cases were stage I-II, III and IV, respectively; while 10 cases from normal esophageal mucosa were used as a control group. With regards to cancer cell differentiation, 10, 16 and 22 cases were well-, moderately and poorly differentiated, respectively.

Treatment methods. Paclitaxel $\left(180 \mathrm{mg} / \mathrm{m}^{2}\right)$ was infused on day 1 and cisplatin $\left(20 \mathrm{mg} / \mathrm{m}^{2}\right)$ was infused on days $1-3$ of a 21-day cycle, yielding a total of 4 chemotherapy cycles. The patients were also administered conventional anti-allergy pretreatment prior to chemotherapy.

Short-term efficacy evaluation. All patients received an endoscopy examination, esophageal barium X-ray and a neck and chest computed tomography (CT) scan prior to treatment and 1 month following treatment. The evaluation of the response rate was based on WHO treatment efficacy evaluation criteria for solid tumors, which divides efficacy into the subgroups: CR, complete remission; PR, partial remission; $\mathrm{SD}$, no change; $\mathrm{PD}$, progressive disease; $\mathrm{CR}+\mathrm{PR}$, overall response rate (13).

Follow-up cases. During follow-up, all patients were contacted via telephone and the last date of follow-up was March 12010. A total of 3 cases were lost; thus, the follow-up rate was $93.8 \%$. The follow-up time ranged from 6 to 50 months and the average time was 16 months. The survival time was calculated from the diagnostic date of esophageal carcinoma to the date of mortality or the last date of follow-up.

Antibody. The antibody used in this study was rabbit polyclonal antibody anti-human cyclin A (Thermo Fisher Scientific, Fremont, CA, USA). The final diluted concentration for anti-cyclin A in TBS containing $1 \%$ bovine serum albumin (BSA) was 1:100.

Immunohistochemical staining. Sliced sections $(4 \mu \mathrm{m})$ of paraffin-embedded specimens were prepared on microscope slides pre-coated with saline. Once the paraffin was removed by xylene, the slides were washed in a graded series of ethanol and the sections were placed in Tris-buffered saline (TBS) for $10 \mathrm{~min}$. Sections were then incubated with a blocking solution for 5 min to block endogenous peroxidase activity and placed in TBS. Sections were then placed in $0.01 \mathrm{mM}$ Tris buffer ( $\mathrm{pH} \mathrm{6.0)}$ and heated at $121^{\circ} \mathrm{C}$ for $20 \mathrm{~min}$ in an autoclave oven. Following this, the sections were incubated with TBS consisting of $1 \%$ BSA for 20 min to block non-specific binding of the immunoreagents. Once the cells were washed in TBS, the sections were incubated with 1:100 diluted primary antibodies at $4^{\circ} \mathrm{C}$ overnight. Following further washing in TBS, an immunoperoxidase staining was performed by a MaxVision antibody complex method using the MaxVision kit (Fujian
Maixin Biological Technology, Fujian, China). Finally, the localization of cyclin A was visualized using diaminobenzidine tetrahydrochloride (DAB) and the sections were lightly counterstained in Harris' hematoxylin solution for microscopic examination.

The immunostained specimens were analyzed by two independent pathologists. At least 10 visual fields were observed and nuclear staining was used to indicate a positive result. A total of 1,000 cells in the tumor and nontumor sections were evaluated at a medium magnification (x200) to determine the proportion of tumor cells and the staining intensity of the nuclei in entire sections. Immunohistochemical expression of cyclin A was determined according to the $a+b$ criteria (14). Category a was defined as the percentage of carcinoma cells with cyclin A expression. The percentage of positively stained tumor cells was determined semi-quantitatively by assessing the whole tumor section, and each sample was assigned to one of the following categories: 0, 0\% cyclin A expression; $1,1-25 \% ; 2,26-50 \% ; 3,>50 \%$. Category $b$ was defined by the intensity of cyclin A staining. The intensity of immunostaining was defined as 0 (negative), 1 (weak), 2 (moderate) and 3 (strong). The carcinomas were regarded to have a positive response to cyclin A when the total scores of $a+b$ were $>3$.

Statistical analysis. The Chi-square test was used to examine the difference between the two groups, and the Cox proportional hazards model was used to analyze the association of overall survival in cyclin A positive and negative expression groups. Variables significantly associated with survival in univariate Cox models were included in a multivariate Cox model. The results were quantified by calculating the hazard ratios (HRs) with $95 \%$ confidence intervals (CIs). Survival curves were calculated using the Kaplan-Meier method and differences between the curves were determined using the log-rank test.

In all tests, $\mathrm{P}<0.05$ was considered to indicate a statistically significant difference. Statistical calculations were conducted using the SPSS version 16.0 System (SPSS, Chicago, IL, USA).

\section{Results}

Expression pattern of cyclin A in normal human esophageal mucosa and ESCC. Cyclin A was expressed mainly in the nuclei of cancer cells and in the basal cells of normal esophageal mucosa $(\mathrm{P}<0.05$; Fig. 1). The positive cyclin A expression rate was $64.6 \%(31 / 48)$ in 48 cases with ESCC, and 20\% (2/10) in 10 cases with normal esophageal mucosa. Comparisons between cyclin A expression pattern in ESCC and normal tissues have been revealed in our previous studies $(10,11)$.

Cyclin A staining and clinicopathological factors. The correlations between cyclin A expression and clinicopathological factors of ESCC are summarized in Table I. Statistically, the expression of cyclin A was not significantly associated with the age, gender, tumor size or clinical stage of the patients; however, the expression levels of cyclin A were significantly higher in poorly differentiated ESCC cases compared with well-differentiated ESCC cases $\left(\chi^{2}=5.274 ; \mathrm{P}<0.05\right)$. This results is consistent with our previous studies in post-surgery ESCC samples $(10,11)$. 
Table I. Correlation between cyclin A expression and clinicopathological factors.

\begin{tabular}{|c|c|c|c|c|}
\hline $\begin{array}{l}\text { Clinicopathological } \\
\text { factors }\end{array}$ & $\begin{array}{c}\text { Positive }(\%) \\
n=31\end{array}$ & $\begin{array}{l}\text { Negative }(\%) \\
n=17\end{array}$ & $\chi^{2}$ & P-value \\
\hline \multicolumn{5}{|l|}{ Age (years) } \\
\hline$\geq 60$ & $25(71.4)$ & $10(28.6)$ & & \\
\hline$<60$ & $6(46.2)$ & $7(53.8)$ & 2.647 & 0.104 \\
\hline \multicolumn{5}{|l|}{ Gender } \\
\hline Male & $21(63.6)$ & $12(36.4)$ & & \\
\hline Female & $10(66.7)$ & $5(33.3)$ & 0.041 & 0.839 \\
\hline \multicolumn{5}{|l|}{ Tumor size $(\mathrm{cm})$} \\
\hline$<5$ & $14(56.0)$ & $11(44.0)$ & & \\
\hline$\geq 5$ & $17(73.9)$ & $6(26.1)$ & 1.680 & 0.195 \\
\hline \multicolumn{5}{|l|}{ TNM stage } \\
\hline I-III & $12(60.0)$ & $8(40.0)$ & & \\
\hline IV & $19(67.9)$ & $9(32.1)$ & 0.315 & 0.575 \\
\hline \multicolumn{5}{|l|}{ Differentiation } \\
\hline Well/moderate & $13(50.0)$ & $13(50.0)$ & & \\
\hline Poor & $18(81.8)$ & $4(18.2)$ & 5.274 & 0.022 \\
\hline
\end{tabular}

A

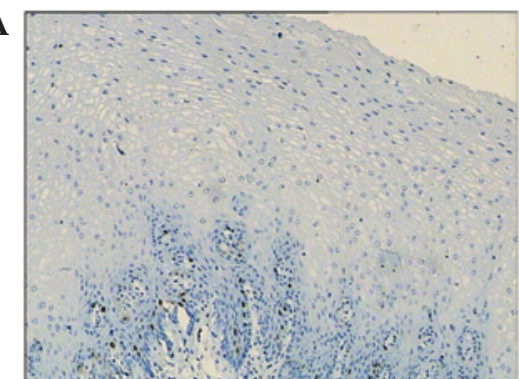

B

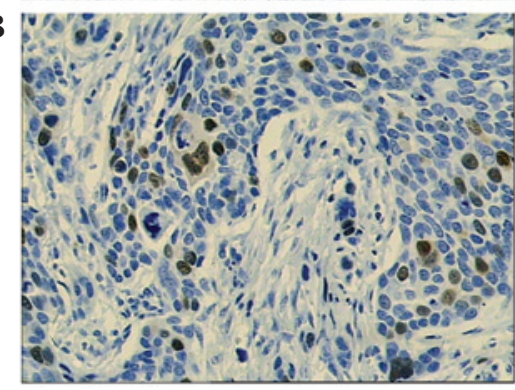

Figure 1. (A) Positive cyclin A expression in normal human esophageal mucosa (MaxVision; magnification, $\mathrm{x} 100$ ) only expressed in basal cells (B) Positive cyclin A expression in ESCC (MaxVision; magnification, $\mathrm{x} 200$ ) expressed mainly in the nuclei of cancer cells. ESCC, esophageal squamous cell carcinoma.

Cyclin A staining and short-term treatment efficacy. Of the 48 ESCC patients, the number of CR, PR, SD and PD cases were $6,15,24$ and 3 , respectively. The overall response rate (CR+PR) was $43.8 \%(21 / 48)$. According to the expression levels of cyclin $\mathrm{A}$, the response rate was $54.8 \%$ (17/31) and $23.5 \%(4 / 17)$ in the positive and negative group, respectively $\left(\chi^{2}=4.373 ; \mathrm{P}=0.037\right.$; Table II).

Cyclin A staining and prognosis. Univariate Cox analysis revealed that the clinicopathological stage, degree of cancer cell differentiation and expression of cyclin A were impact
Table II. Correlation between cyclin A expression and short-term treating efficacy.

\begin{tabular}{lcccc}
\hline Efficacy & $\begin{array}{c}\text { Positive } \\
\mathrm{n}=31(\%)\end{array}$ & $\begin{array}{c}\text { Negative } \\
\mathrm{n}=17(\%)\end{array}$ & $\chi^{2}$ & P-value \\
\hline $\mathrm{CR}$ & $5(16.13)$ & $1(5.88)$ & 1.054 & 0.305 \\
$\mathrm{PR}$ & $12(38.71)$ & $3(17.65)$ & 2.267 & 0.132 \\
$\mathrm{SD}$ & $13(41.94)$ & $11(64.71)$ & 0.024 & 0.877 \\
$\mathrm{PD}$ & $1(3.22)$ & $2(11.76)$ & 4.296 & 0.039 \\
CR+PR & $17(54.84)$ & $4(23.53)$ & 4.373 & 0.037 \\
\hline
\end{tabular}

${ }^{\mathrm{a} O v e r a l l ~ r e s p o n s e ~ r a t e . ~} \mathrm{CR}$, complete remission; PR, partial remission; $\mathrm{SD}$, no change; $\mathrm{PD}$, progressive disease.

factors for prognosis in ESCC patients; while no significant correlation was observed between age, gender and tumor size (Table III). The median survival time for patients with positive cyclin A expression was 17 months. This was significantly shorter compared with that for the patients with negative cyclin A expression, which was 33 months $(\mathrm{P}=0.016)$. Kaplan-Meier survival curves demonstrated that the 1- and 3-year survival rates in patients with positive cyclin A expression were significantly lower compared with that of the patients with negative cyclin A expression (54.9 vs. 82.4\%, $\mathrm{P}=0.037$; 6.2 vs. $41.2 \%, \mathrm{P}=0.016$, respectively) (Fig. 2).

Multivariate Cox analysis revealed that the clinicopathological stage, degree of cancer cell differentiation and expression of cyclin A were of statistical significance in ESCC patients. We demonstrated that advanced clinicopathological stage $(\mathrm{P}=0.000 ; 95 \% \mathrm{CI}, 0.018-0.309)$, poor cancer cell differentiation $(\mathrm{P}=0.008 ; 95 \% \mathrm{CI}, 0.138-0.739)$ and positive expression of cyclin A ( $\mathrm{P}=0.002,95 \% \mathrm{CI}, 0.119-0.611)$ were independent prognostic factors in patients with ESCC following paclitaxel-based chemoradiotherapy (Table IV). 
Table III. Univariate analysis of prognostic impact factors of ESCC patients.

\begin{tabular}{lcccc}
\hline Impact factor & No. & $\begin{array}{c}\text { Median survival } \\
\text { (months) }\end{array}$ & $\chi^{2}$ & P-value \\
\hline Age (years) & & & & \\
$\geq 60$ & 35 & 18 & & \\
$>60$ & 13 & 20 & 0.080 & 0.778 \\
Gender & & & & \\
$\quad$ Male & 33 & 18 & & \\
$\quad$ Female & 15 & 25 & 0.076 & 0.783 \\
$\quad$ umor size (cm) & & & & \\
$\quad<5$ & 25 & 20 & & \\
$\geq 5$ & 23 & 18 & 0.116 & 0.733 \\
$\quad$ TNM stage & & & & \\
I-III & 20 & 28 & & \\
IV & 28 & 10.5 & 3.980 & 0.046 \\
Differentiation & & & & \\
$\quad$ Well/moderate & 26 & 26 & & \\
$\quad$ Poor & 22 & 12 & 5.863 & 0.015 \\
Cyclin A & & & & \\
expression & & & & \\
$\quad$ Positive & 31 & 17 & & \\
$\quad$ Negative & 17 & 33 & 5.749 & 0.016 \\
\hline
\end{tabular}

ESCC, esophageal squamous cell carcinoma.

\section{Discussion}

Since the overall survival rate of ESCC patients following surgery alone is poor, multimodality approaches have been developed. However, these are inadequate with regard to improving the treatment efficacy of esophageal cancer by basing treatment on surgical approaches alone. Instead, surgeons should be prepared for a new approach, which comprises biological tumor staging and targeted therapies combined with neoadjuvant chemoradiotherapy. Certain studies suggest that the tumor response to induction chemoradiotherapy aids the identification of patients with good prognosis, regardless of whether surgery is conducted or not $(3,15)$. In these patients, surgery may no longer be recommended as routine treatment (15); therefore, ESCC patients may have a survival benefit if they are responsive to chemoradiotherapy. To predict

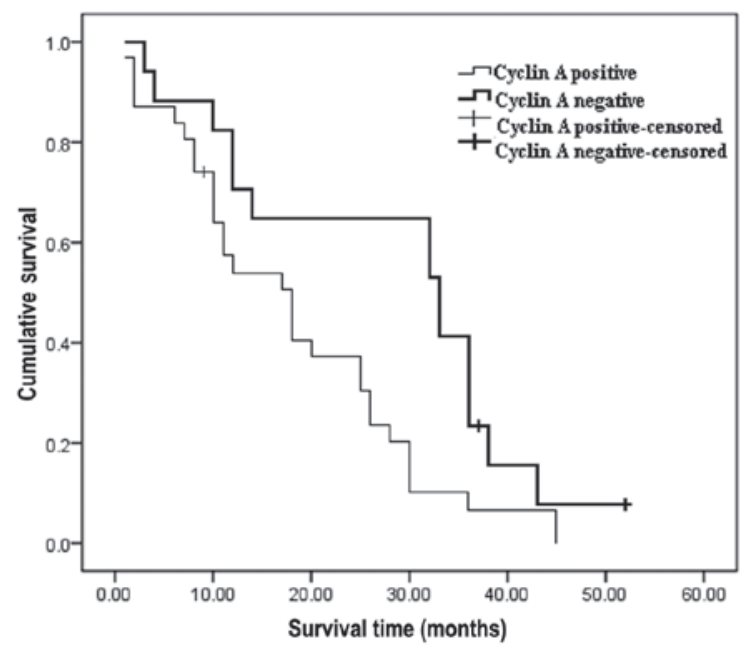

Figure 2. Kaplan-Meier survival curve reveals that patients with positive cyclin A expression have a lower survival rate.

the response prior to chemoradiotherapy is undoubtedly one of the critical factors to determine the best treatment. Although not all tumors respond to paclitaxel, it is used widely in the treatment of ESCC. A previous study has revealed that the response rate of paclitaxel as a single drug in ESCC patients was only $20-37 \%$, and the overall response rate of paclitaxel in combination with cisplatin was $42.6 \%$ (16). The characteristics that distinguish drug-resistant tumors from drug-sensitive tumors are not well defined, and for that reason, predicting the response prior to chemotherapy is a research hotspot. Therefore, several studies have focused on predicting paclitaxel sensitivity in vitro using BRCA1, CDK1 and CDK2 $(17,18)$.

Cyclins, which control key checkpoints of the cell cycle, play a fundamental role in regulating cell growth and survival. In humans, several types of cyclin, including three categories, A-, B- and C-type (C-, D- and E-types), have now been isolated. Cyclins A and B1-2 reach maximum levels in the S and G2 phases. Cyclin A may be activated during the transition from the G1 to the S phase of the cell cycle; therefore, it is suggested to be an important regulator of mitosis $(19,20)$. In this study, we detected the expression of cyclin A in tissue samples from 48 patients with ESCC prior to treatment with paclitaxel/cisplatin. We revealed that the expression of cyclin A was significantly correlated with the degree of cell differentiation and the response to paclitaxel-based chemotherapy. The expression of cyclin A in the poorly differentiated ESCC samples was higher compared with that of the well-differ-

Table IV. Multivariate Cox analysis of clinicopathological and biological molecular characteristics in ESCC patients.

\begin{tabular}{lccccccr}
\hline & & & & & & \multicolumn{2}{c}{$95 \%$ CI for Exp (B) } \\
\cline { 5 - 8 } & B & SE & Wald & P-value & Exp (B) & Lower & Upper \\
\hline TNM stage & -2.607 & 0.732 & 12.693 & 0.000 & 0.074 & 0.018 & 0.309 \\
Differentiation & -1.414 & 0.427 & 7.133 & 0.008 & 0.320 & 0.138 & 0.739 \\
Cyclin A expression & -1.312 & 0.418 & 9.845 & 0.002 & 0.269 & 0.119 & 0.611 \\
\hline
\end{tabular}

ESCC, esophageal squamous cell carcinoma; CI, confidence interval. 
entiated ESCC samples, indicating that cyclin A expression correlates with the degree of ESCC malignancy. This suggests that ESCC samples with positive cyclin A expression were poorly differentiated, and the cell proliferation cycle was more active. Of the 48 cases of ESCC, the response rate of 31 cases with positive cyclin A expression was $54.8 \%$, while the response rate of 17 cases with negative cyclin A expression was $23.5 \%$. This result indicates that ESCC patients with positive cyclin A expression were sensitive to paclitaxel-based chemotherapy and may have a higher response rate.

Paclitaxel binds to microtubules and stabilizes the polymerized structure. Consequently, paclitaxel inhibits microtubule depolymerization, suppressing tumor growth and holding proliferative cells in the $\mathrm{G} 2 / \mathrm{M}$ phase (21). Nakayama et al reported that the analysis of cyclin-dependent kinase activity in the clinical setting may be a powerful approach for predicting paclitaxel sensitivity (18). Another study revealed that targeting cyclin B1 sensitized breast cancer cells to taxol, suggesting that specific cyclin B1 targeting is an attractive strategy to be used in combination with conventionally used agents in gynecological cancer therapy (22). Our study demonstrated that cyclin A expression was correlated with chemosensitivity to paclitaxel-based chemotherapy in patients with ESCC. This indicates that the cell cycle is located at the G2/M phase and that when the expression of cyclin A was positive in tumor cells, patients had a higher response to paclitaxel, which is consistent with the cell cycle theory.

Our data also revealed that ESCC patients with positive cyclin A expression had poor prognosis, while the patients with negative cyclin A expression had good prognosis. This result is consistent with previous studies on breast (23), ovarian (24) and laryngeal cancer (25).

Determining whether the tumor is sensitive to paclitaxel prior to treatment is one of the most efficient ways of improving response. Our study revealed that ESCC patients overexpressing cyclin A prior to treatment may be more sensitive to paclitaxel-based chemotherapy. It would be beneficial to extend this preliminary result into a larger sample size study in order to formalize it as a standard procedure for the clinical treatment of ESCC. Our study indicates the importance of applying 'personalized cancer medicine' in the clinical treatment of cancer, which is based on gene expression profile, to guide the antitumor drug selection.

\section{Acknowledgements}

This study was supported by the 333 Plan Foundation (Grant No. 2009-24) of Jiangsu Province, China, the Six Talents Peak Foundation (Grant No. 2011-WS-023) of Jiangsu, China, and the Key Medical Talent Foundation (Grant No. RC2011212) of Jiangsu, China. The authors are grateful to Dr Jianming Zhang for his assistance with the manuscript preparation.

\section{References}

1. Enzinger PC and Mayer RJ: Esophageal cancer. N Engl J Med 349: 2241-2252, 2003.

2. Takeno S, Noguchi T, Takahashi Y, Fumoto S, Shibata T and Kawahara K: Assessment of clinical outcome in patients with esophageal squamous cell carcinoma using TNM classification score and molecular biological classification. Ann Surg Oncol 14: 1431-1438, 2007.
3. Ilson DH: Esophageal cancer chemotherapy: recent advances. Gastrointest Cancer Res 2: 85-92, 2008.

4. Bedenne L, Michel P, Bouché O, et al: Chemoradiation followed by surgery compared with chemoradiation alone in squamous cancer of the esophagus: FFCD 9102. J Clin Oncol 25: 1160-1168, 2007.

5. Abrams JA, Buono DL, Strauss J, McBride RB, Hershman DL and Neugut AI: Esophagectomy compared with chemoradiation for early stage esophageal cancer in the elderly. Cancer 115: 4924-4933, 2009.

6. Yamashita H, Okuma K, Seto Y, et al: A retrospective comparison of clinical outcomes and quality of life measures between definitive chemoradiation alone and radical surgery for clinical stage II-III esophageal carcinoma. J Surg Oncol 100: 435-441, 2009.

7. Ariga H, Nemoto K, Miyazaki S, et al: Prospective comparison of surgery alone and chemoradiotherapy with selective surgery in resectable squamous cell carcinoma of the esophagus. Int J Radiat Oncol Biol Phys 75: 348-356, 2009.

8. Cordon-Cardo C: Mutations of cell cycle regulators. Biological and clinical implications for human neoplasia. Am J Pathol 147: 545-560, 1995.

9. Huang JX, Yan W, Song ZX, et al: Relationship between proliferative activity of cancer cells and clinicopathological factors in patients with esophageal squamous cell carcinoma. World $\mathrm{J}$ Gastroenterol 11: 2956-2959, 2005.

10. Huang JX, Xiao W, Chen WC, et al: Relationship between COX-2 and cell cycle-regulatory proteins in patients with esophageal squamous cell carcinoma. World J Gastroenterol 16: 5975-5981, 2010.

11. Huang JX, Chen WC, Lin M, et al: Clinicopathological significance of cyclooxygenase-2 and cell cycle-regulatory proteins expression in patients with esophageal squamous cell carcinoma. Dis Esophagus 25: 121-129, 2012.

12. Huang J: Esophageal cancer In: Manual Medical Oncology. Sun Y and Shi YK (eds). 5th edition. The People's Medical Publishing House, Beijing, pp466-469, 2007.

13. Sun Y: The clinical experiment of anti-tumor drugs In: Manual Medical Oncology. Sun Y and Shi YK (eds). 5th edition. The People's Medical Publishing House, Beijing, pp161-162, 2007.

14. Nozoe T, Korenaga D, Futatsugi M, Saeki H, Ohga T and Sugimachi K: Cyclin A expression in superficial squamous cell carcinoma of the esophagus and coexisting infiltrated lymphocyte follicle. Cancer Lett 188: 221-229, 2002.

15. Wolf MC, Stahl M, Krause BJ, et al: Curative treatment of oesophageal carcinoma: current options and future developments. Radiat Oncol 6: 55, 2011.

16. Zhang XD, Shen L, Li J, et al: Prospective non-randomized study of chemotherapy combined with radiotherapy versus chemotherapy alone in patients with metastatic or relapsed esophageal squamous cell carcinoma. Zhonghua Zhong Liu Za Zhi 29: 474-477, 2007 (In Chinese).

17. Stordal B and Davey R: A systematic review of genes involved in the inverse resistance relationship between cisplatin and paclitaxel chemotherapy: role of BRCA1. Curr Cancer Drug Targets 9: 354-365, 2009.

18. Nakayama S, Torikoshi Y, Takahashi T, et al: Prediction of paclitaxel sensitivity by CDK 1 and CDK 2 activity in human breast cancer cells. Breast Cancer Res 11: R12, 2009.

19. Hunter T and Pines J: Cyclins and cancer. Cell 66: 1071-1074, 1991.

20. Pagano M, Pepperkok R, Verde F, Ansorge W and Draetta G: Cyclin A is required at two points in the human cell cycle. EMBO J 11: 961-971,1992.

21. Mitchison TJ: The proliferation rate paradox in antimitotic chemotherapy. Mol Biol Cell 23: 1-6, 2012.

22. Androic I, Krämer A, Yan R, et al: Targeting cyclin B1 inhibits proliferation and sensitizes breast cancer cells to taxol. BMC Cancer 8: 391-402, 2008

23. Boström P, Söderström M, Palokangas T, Vahlberg T, Collan Y, Carpen $\mathrm{O}$ and Hirsimäki P: Analysis of cyclins A, B1, D1 and E in breast cancer in relation to tumour grade and other prognostic factors. BMC Res Notes 2: 140-148, 2009.

24. Yoon BS, Kim YT, Kim S, et al: Prognostic value of nuclear DNA quantification and cyclin A expression in epithelial ovarian carcinoma. Eur J Obstet Gynecol Reprod Biol 136: 110-115, 2008.

25. Fraczek M, Wozniak Z, Ramsey D, Zatonski T and Krecicki T: Clinicopathologic significance and prognostic role of cyclin $\mathrm{E}$ and cyclin A expression in laryngeal epithelial lesions. Acta Otolaryngol 128: 329-334, 2008. 